\title{
A common 56-kilobase deletion in a primate-specific segmental duplication creates a novel butyrophilin-like protein
}

Johanna Aigner 1,2,3,4 Sergi Villatoro 1,2,3,4, Raquel Rabionet ${ }^{1,2,3,4}$, Jaume Roquer ${ }^{5,6}$, Jordi Jiménez-Conde ${ }^{5,6}$, Eulàlia Marti ${ }^{1,2,3,4}$ and Xavier Estivill ${ }^{1,2,3,4^{*}}$

\begin{abstract}
Background: The Butyrophilin-like (BTNL) proteins are likely to play an important role in inflammation and immune response. Like the B7 protein family, many human and murine BTNL members have been shown to control T lymphocytes response, and polymorphisms in human BTNL2 have been linked to several inflammatory diseases, such as pulmonary sarcoidosis, inflammatory bowel disease and neonatal lupus.

Results: In this study we provide a comprehensive population, genomic and transcriptomic analysis of a 56-kb deletion copy number variant (CNV), located within two segmental duplications of two genes belonging to the BTNL family, namely BTNL8 and BTNL3. We confirm the presence of a novel BTNL8*3 fusion-protein product, and show an influence of the deletion variant on the expression level of several genes involved in immune function, including BTNL9, another member of the same family. Moreover, by genotyping HapMap and human diversity panel (HGDP) samples, we demonstrate a clear difference in the stratification of the BTNL8_BTNL3-del allele frequency between major continental human populations.

Conclusion: Despite tremendous progress in the field of structural variation, rather few CNVs have been functionally characterized so far. Here, we show clear functional consequences of a new deletion CNV (BTNL8_BTNL3-del) with potentially important implication in the human immune system and in inflammatory and proliferative disorders. In addition, the marked population differences found of BTNL8_BTNL3-del frequencies suggest that this deletion CNV might have evolved under positive selection due to environmental conditions in some populations, with potential phenotypic consequences.
\end{abstract}

\section{Background}

The human genome has been shown to be quite plastic, with many regions presenting gains and losses of genetic material amongst individuals, also known as copy number variants (CNVs) [1]. Both germline and somatic CNVs have been found to play important roles in several disorders, including neuropsychiatric, infectious and autoimmune diseases, and cancer [2-5]. In addition, variation in copy number has been shown to be a major driving force in evolution, especially within the primate lineage. Compared to other mammals, such as rat and

\footnotetext{
* Correspondence: xavier.estivill@crg.eu

'Bioinformatics and Genomics Program, Centre for Genomic Regulation (CRG), Barcelona 08003, Spain

¿Universitat Pompeu Fabra (UPF), Barcelona 08003, Spain

Full list of author information is available at the end of the article
}

mouse, the genome of humans and great ape species is characterized by a significant enrichment in CNVs [6]. A high percentage of these variants (25\%-50\%) are located in close proximity to or are part of segmental duplications (SDs), also called low-copy repeat (LCRs) elements, which present blocks of highly $(>95 \%)$ identical sequences generated during primate evolution $[7,8]$. Regions enriched in LCRs predispose to genomic rearrangement during meiosis, originated by non-allelic homologous recombination (NAHR) between repetitive LCR elements [9]. Interestingly, CNVs that overlap with SDs have been shown to be especially rich in gene and pseudogene content, and therefore are likely to be of clinical importance. Moreover, genes with functions related to immunity and infection are enriched in CNVs, and most primate-specific hotspots for CNVs formation have been found in

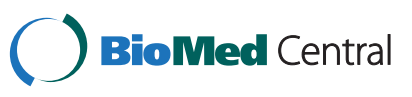


genes with roles in immune or environmental response, suggesting an association between CNVs and SDs in human health $[10,11]$.

The precise structure of many CNVs and their potential functional consequences are still largely unknown. In the present study, by using lymphoblastoid cell lines (LCL) derived from human subjects of European ancestry, we undertook a systematic genetic, gene expression and evolutionary analysis of a previously uncharacterized 56-kb deletion $\mathrm{CNV}$, located on the subtelomeric region of human chromosome 5q35.3, in a cluster with several genes encoding tripartite motif-containing (TRIM) proteins and genes involved in the olfactory system. We show that the breakpoints of the deletion are located within two primatespecific SDs of two genes belonging to the butyrophilinlike (BTNL) protein family, and that the polymorphic deletion (BTNL8_BTNL3-del allele) leads to the formation of a new fusion gene (BTNL8*3). We confirm the presence of a novel BTNL8*3 fusion-protein product, and show an influence of the deletion variant on the expression level of neighbouring gene BTNL9 and several other genes involved in immune response and cancer, thus suggesting an involvement of this CNV in specific biological pathways. Moreover, we have found differences in the frequency of the deletion allele amongst major continental ethnic groups, being rare in African and Oceanic populations but common in Asians, Americans and Europeans. After carefully looking for tagging single nucleotide polymorphism (SNPs) that could be used as a surrogate for the deletion, we only were able to identify a suitable tag SNP in some populations tested, suggesting that the $\mathrm{CNV}$ is a recent and recurrent event in humans. Taken together, our findings show functional consequences of a novel deletion polymorphism with impact in world population's distribution and potential implications in physiological processes of immune response and proliferation.

\section{Results \\ Array-CGH analysis of chromosome 5 q35.3 reveals a 56-kb polymorphic deletion}

By using the human Agilent 244 K whole-genome array and consequent customized Multiplex Ligation-dependent Probe Amplification (MLPA) analysis of LCLs derived from HapMap samples, we found a potential CNV, located on chromosome 5q35.3. We next developed a direct PCR amplification assay to map the breakpoints of the CNV, finding that it consists of a $\sim 56-\mathrm{kb}$ deletion polymorphism (chr5:180375027-180430596 in hg19) recently reported by $\mathrm{Kim}$ et al. [12]. The $\mathrm{CNV}$ removes the genomic sequence between intron four of BTNL8 and intron four of $B T N L 3$, resulting in a new $B T N L 8 * 3$ fusion gene without any alterations in the coding sequence (Figure 1A). The breakpoints of the deleted allele are located in two $1.6-\mathrm{kb}$ SD that share $98 \%$ identity and are separated by $~ 55,570$ nucleotides on the BTNL8 and BTNL3 genes, indicating that the deletion resulted from a NAHR event. Each 1.6-kb SD contains parts of intron four to exon eight of BTNL8 and BTNL3. The crossover occurred somewhere within 112-bp of identical sequence of these two genes in intron four (Figure 1B).

\section{Linkage disequilibrium with SNPs in the genomic region}

To evaluate linkage disequilibrium (LD) between BTNL8_BTNL3-del and tag single nucleotide polymorphisms (SNPs) covering the BTNL8 and BTNL3, surrounding haplotype structure, we genotyped the $56-\mathrm{kb}$ deletion in 1,103 unrelated individuals included in the HapMap Phase III project and searched for potential tagging SNPs, based on the genotypes on the HapMap data release 28 (http://hapmap.ncbi.nlm.nih.gov), and an independent control cohort of 2,000 Spanish unrelated individuals. Several SNPs were in high LD $\left(\mathrm{r}^{2}>0.9\right)$ with the deletion in the CEU, CHD, CHB, MEX, GHI and JPT sets. The best taggers in these populations were rs2387715 and rs4700772 (CEU, CHB, JPT, $\mathrm{r}^{2}=1$; MEX, $\mathrm{r}^{2}=0.95$; CHD, $r^{2}=0.96 ;$ and GHI, $\left.r^{2}=0.94\right)$. Finally, in the Tuscan population only one SNP was selected as a tagger, rs10063135 (TSI, $r^{2}=0.81$ ). In contrast, we could not identify a proxy SNP $\left(\mathrm{r}^{2}>0.8\right)$ in African-ancestry populations (ASW, LWK, MKK or YRI). In fact, the SNPs in higher LD in these populations were: $r s 3733755$ (YRI, $\mathrm{r}^{2}=$ 0.38 ); rs2387715 (ASW, $\mathrm{r}^{2}=0.69 ; \mathrm{MKK}, \mathrm{r}^{2}=0.32$ ); and rs4701016 (LWK, $r^{2}=0.36$ ) (Table 1). Based on these LD results, we selected three of these SNPs (rs3733755, rs10063135 and rs4700772) for genotyping 2,000 Spanish samples, and none of them showed strong LD $\left(r^{2}>0.8\right)$ with the deletion in our dataset: rs10063135, $r^{2}=0.63 ; r s 3733755, r^{2}=0.63$, and $r s 4700772 r^{2}=0.724$.

\section{Population differences in the frequency of the BTNL8_BTNL3-del allele}

To check whether population-based differences exist for the BTNL8_BTNL3-del allele, HapMap samples from 120 CEU; 90 CHB; 90 JPT; 120 YRI; 43 ASW; 100 CHD; 100 GIH; 100 LWK; 90 MEX; 150 MKK; 100 TSI; and 2,000 Spanish samples were genotyped. In addition, we analyzed 1,007 samples from 39 ethnical groups of the Centre d'Etude du Polymorphisme Humain (CEPH) Human Genome Diversity Panel (HGDP) (Additional file 1: Table S1 and Table S2).

We observed clear differences in allele frequencies between major continental groups. The deletion is significantly underrepresented in Oceanic, central Asian and Sub-Saharan African populations but significantly overrepresented in European and American populations. In CEU population, $12 \%$ are homozygous for the deletion allele and $41 \%$ carry at least one BTNL8_BTNL3-del allele, corresponding to an allele frequency of $34 \%$. In the CHBJPT population the deleted allele shows a frequency of 


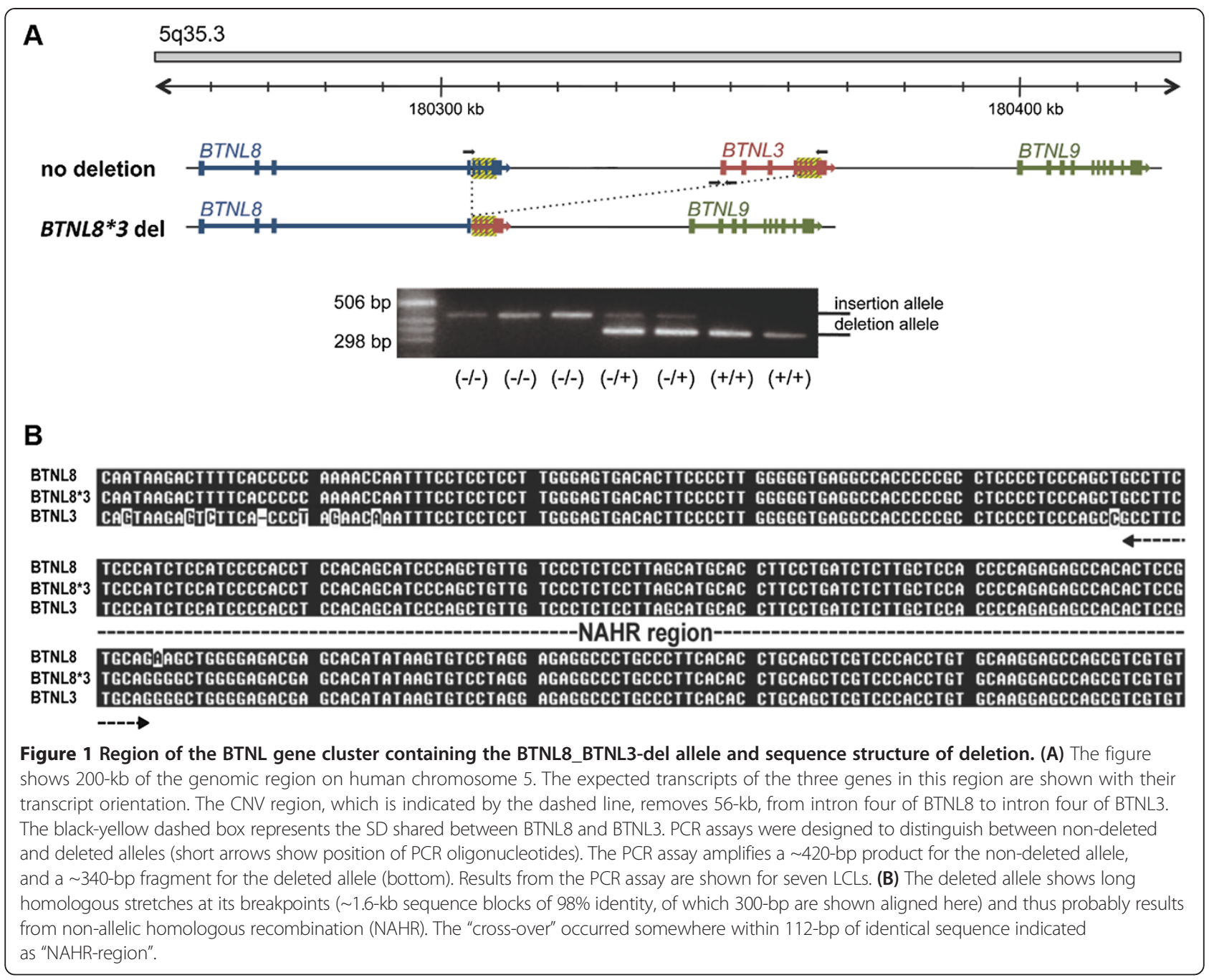

29\%, while in African and Oceanic populations show a significantly reduced frequency for the BTNL8_BTNL3-del allele of $9 \%$ and $3 \%$, respectively (Figure 2, Additional file 1: Table S3). In order to rule out large-scale genotyping errors, we calculated estimates of Hardy-Weinberg equilibrium for each of the 39 HGDP populations, as well as for all continental HapMap and HGDP groups, but could not observe any significant deviations except for Hezhen (China), Cambodian and Orcadian population, which most likely is due to the small sample size (10, 10 and 15 individuals, respectively) sequenced (Additional file 1: Table S3 and Table S4).

The BTNL8*3 56-kb deletion leads to a new chimeric transcript and protein product

To further analyse the CNV at the transcriptional level, LCLs were genotyped and RT-PCR analysis and subsequent sequencing was carried out on samples containing none, one or two copies of the BTNL8_BTNL3-del allele. In cell lines heterozygous and homozygous for the deletion $\mathrm{CNV}$ we detected a new, in-frame, fusion transcript, $B T N L 8 * 3$, which consisted of the juxtaposition of exons one to four of BTNL8 to exons five to eight of $B T N L 3$ (Figure 3A). Thus, the predicted BTNL8*3 transcript would contain the 3'-UTR from BTNL3, but the $B T N L 8$ upstream regulatory signals. In a next step, allelespecific quantitative RT-PCR (qPCR) analysis was performed on LCL and tissue samples heterozygous for the BTNL8_BTNL3-del allele. A chimeric BTNL8*3 transcript could be identified in all samples (Figure $3 \mathrm{~B}$ ). However, the fusion transcript was present at a significantly reduced level, compared to BTNL 8 mRNA with which it shares its complete upstream regulatory region. Moreover, western-blot analysis revealed the presence of a novel BTNL8*3 fusion protein in LCLs carrying the deleted allele, indicating the presence of a novel protein (Figure 3C). BTNL8 and BTNL3 share $68.5 \%$ similarity in their protein sequences (Additional file 1: Figure S1). The resulting 
Table 1 List of SNPs tested for LD with the BTNL8*3 deletion CNV

\begin{tabular}{|c|c|c|c|c|c|c|c|c|c|c|c|c|c|c|c|c|c|c|c|c|c|c|c|c|}
\hline \multirow[b]{2}{*}{ rs Number } & \multirow[b]{2}{*}{ Position } & \multirow[b]{2}{*}{$\begin{array}{l}\text { Distance to } \\
\text { deletion } \\
\text { breakpoints }\end{array}$} & \multicolumn{2}{|c|}{ ASW } & \multicolumn{2}{|l|}{ CEU } & \multicolumn{2}{|l|}{ CHB } & \multicolumn{2}{|c|}{ CHD } & \multicolumn{2}{|l|}{ GHI } & \multicolumn{2}{|c|}{ JPT } & \multicolumn{2}{|l|}{ LWK } & \multicolumn{2}{|l|}{ MKK } & \multicolumn{2}{|l|}{ MEX } & \multicolumn{2}{|l|}{ TSI } & \multicolumn{2}{|l|}{ YRI } \\
\hline & & & $D^{\prime}$ & $r^{2}$ & $D^{\prime}$ & $r^{2}$ & $D^{\prime}$ & $r^{2}$ & $D^{\prime}$ & $r^{2}$ & $D^{\prime}$ & $r^{2}$ & $D^{\prime}$ & $r^{2}$ & $D^{\prime}$ & $r^{2}$ & $D^{\prime}$ & $r^{2}$ & $D^{\prime}$ & $r^{2}$ & $D^{\prime}$ & $r^{2}$ & $D^{\prime}$ & $r^{2}$ \\
\hline rs1904435 & 180322735 & 52,292 & & & & & 0.94 & 0.834 & 1 & 0.77 & 0.81 & 0.596 & 1 & 0.829 & 0.31 & 0.03 & & & 0.93 & 0.537 & 0.8 & 0.495 & 0.69 & 0.099 \\
\hline rs7735361 & 180329359 & 45,668 & 1 & 0.086 & 0.91 & 0.843 & 1 & 0.658 & 1 & 0.706 & 0.94 & 0.663 & 1 & 0.796 & 0.3 & 0.034 & 0.37 & 0 & 0.82 & 0.58 & 0.8 & 0.54 & 0.03 & 0 \\
\hline rs17704291 & 180336849 & 38,178 & 0.8 & 0.43 & 1 & 0.96 & 0.94 & 0.889 & - & - & - & - & 1 & 1 & 0.28 & 0.04 & & & 0.94 & 0.76 & 0.9 & 0.68 & 0.3 & 0.06 \\
\hline rs4700772 & 180341845 & 33,182 & 0.8 & 0.539 & 1 & 1 & 1 & 1 & 1 & 0.966 & 1 & 0.946 & 1 & 1 & 0.8 & 0.25 & 0.63 & 0.322 & 1 & 0.952 & 1 & 0.752 & 0.8 & 0.3 \\
\hline rs2387715 & 180361266 & 13,761 & 0.8 & 0.694 & 1 & 1 & 1 & 1 & 1 & 0.966 & 1 & 0.944 & 1 & 1 & 0.8 & 0.237 & 0.63 & 0.322 & 1 & 0.952 & 1 & 0.749 & 0.8 & 0.33 \\
\hline rs3733755 & 180374484 & 543 & 1 & 0.686 & 1 & 0.815 & 1 & 0.692 & 1 & 0.451 & 1 & 0.588 & 1 & 0.486 & 1 & 0.31 & 0.77 & 0.195 & 1 & 0.86 & 1 & 0.598 & 1 & 0.38 \\
\hline deletion & $\begin{array}{l}180375027- \\
180430596\end{array}$ & & & & & & & & & & & & & & & & & & & & & & & \\
\hline rs10063135 & 180432024 & 1,428 & 1 & 0.177 & 1 & 0.923 & 1 & 0.946 & 1 & 0.966 & 1 & 0.545 & 1 & 0.754 & 0.4 & 0.161 & 0.8 & 0.101 & 1 & 0.82 & 0.97 & 0.81 & 0.8 & 0.09 \\
\hline rs4700774 & 180434216 & 3,620 & 1 & 0.473 & 1 & 1 & 1 & 0.945 & 1 & 0.966 & 1 & 0.893 & 1 & 1 & 0.84 & 0.12 & 0.5 & 0.09 & 0.95 & 0.903 & 1 & 0.752 & 0.2 & 0.07 \\
\hline rs7721042 & 180439488 & 8,892 & & & 1 & 0.958 & 1 & 0.939 & - & - & - & - & 1 & 1 & - & - & & & & & - & - & 0.03 & 0 \\
\hline rs11249756 & 180455372 & 24,776 & 1 & 0.473 & 1 & 0.923 & 1 & 0.945 & 0.96 & 0.86 & 0.8 & 0.64 & 1 & 1 & 0.4 & 0.161 & 0.44 & 0.07 & 0.94 & 0.807 & 0.94 & 0.72 & 0.2 & 0.07 \\
\hline rs4701016 & 180458539 & 27,943 & 1 & 0.686 & 0.91 & 0.733 & 1 & 0.646 & 0.86 & 0.37 & 0.6 & 0.3 & 1 & 0.486 & 1 & 0.364 & 0.2 & 0.01 & 0.85 & 0.719 & 0.9 & 0.55 & 1 & 0.3 \\
\hline rs6868418 & 180458813 & 28,217 & 1 & 0.686 & 0.91 & 0.733 & 1 & 0.646 & 0.86 & 0.4 & 0.6 & 0.3 & 1 & 0.486 & 1 & 0.364 & - & - & - & - & - & - & 1 & 0.3 \\
\hline
\end{tabular}

HapMap phase III populations were screened for tagging SNPs for the BTNL8_BTNL3-del allele. Several SNPs could be identified in Eastern Asian (CHB, CHD and JPT), American (GIH and MEX) and CEU population. However, no LD could be observed in African population (ASW, LKW, MKK and YRI) and only a weak association for rs 10063135 could be detected in TSI population.

$r^{2}$ higher than0.8 indicates a significant correlation. ASW, individuals with African ancestry from Southwest USA; CEU, individuals from Utah with northern and western European ancestry; CHB, Han Chinese from Bejing; CHD, Han Chinese from Denver, Colorado; GIH, Gujarati Indians from Houston, Texas; JPT, Japanese from Tokyo; LWK, Luhya from Webuya, Kenya; MEK, individuals with Mexican ancestry from Los Angeles, California; MKK, Maasai from Kinyawa, Kenya; TSI, Toscans from Italy; YRI, Yorubas from Ibadan, Nigeria. 


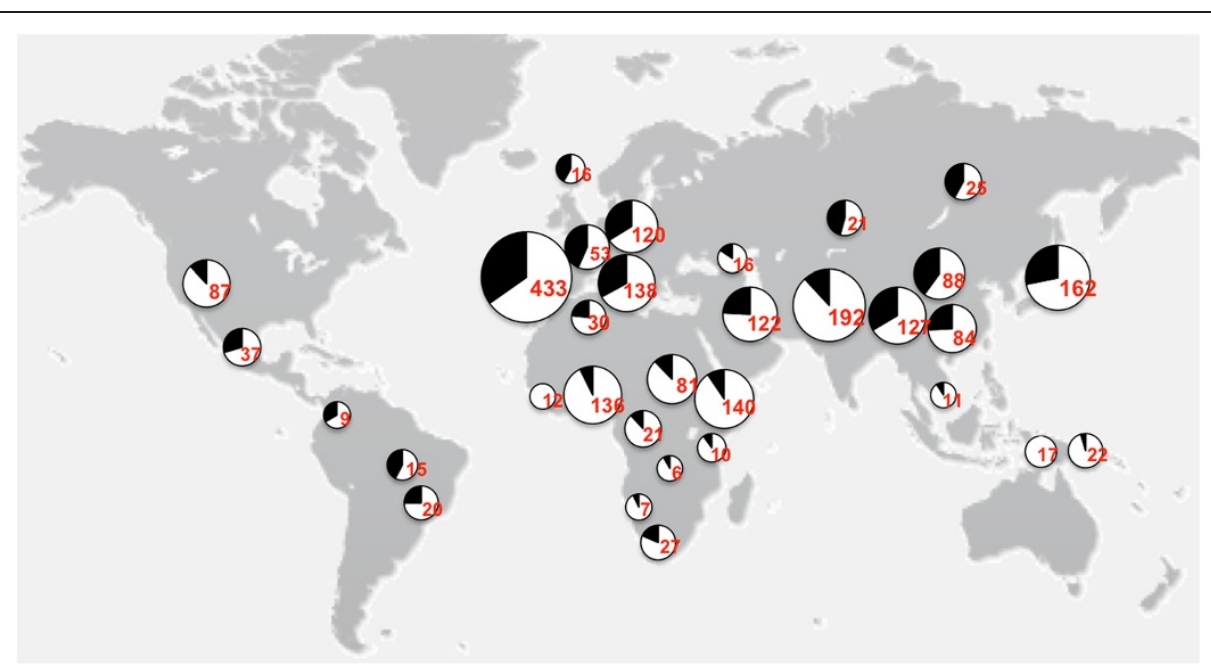

Figure 2 World-wide distribution of the BTNL8_BTNL3-del allele in different human populations. Genotype analysis of populations is shown over a map of the world. The deletion frequency is indicated by the black part of the pie in the chart. Total number of individuals genotyped is given in red.

chimeric protein would contain the extracellular Ig-like C and Ig-like $\mathrm{V}$ domain, and the transmembrane domain of BTNL8 and the intracellular B30.2 domain at its Cterminus (Figure 3D).

\section{$B T N L 8 * 3$ CNV affects expression of neighboring}

\section{gene BTNL9}

$B T N L 8$ and BTNL3 form a cluster together with another gene of the same family, BTNL9. Both, the BTNL9 gene and its promoter region are intact in the BTNL8_BTNL3del allele, thus BTNL9 expression would not be expected to be affected by the BTNL8*3 deletion CNV. However, it is known that genomic neighborhoods may influence the expression level of a gene by a positional effect. This can be achieved by affecting cis-regulatory elements, such as transcription factor binding sites, or by re-organization of chromosomes into territories within the nucleus [13,14]. To test whether this is the case for BTNL9 and other

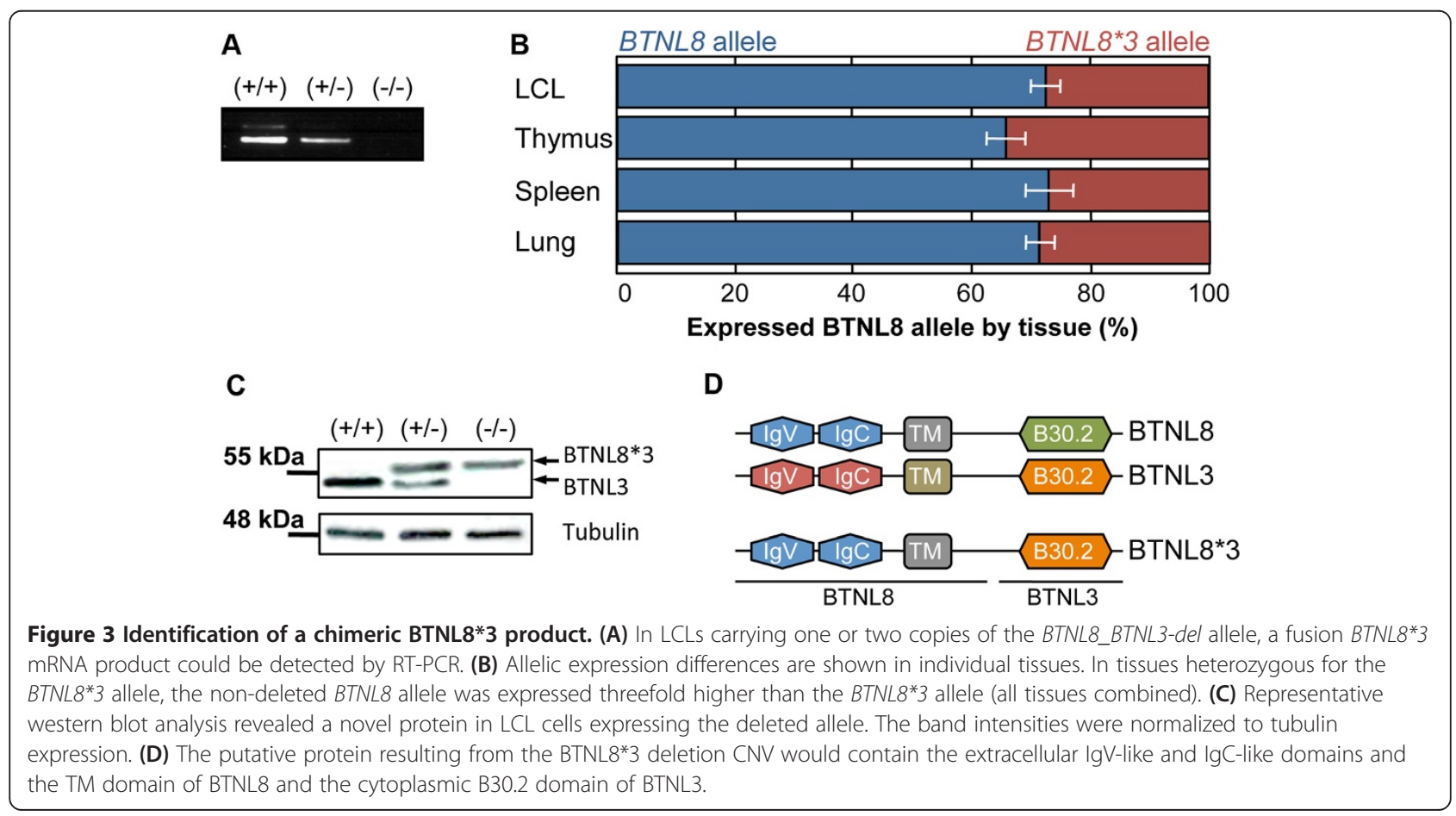


genes located behind the deletion $\mathrm{CNV}$, we measured BTNL9, TRIM7 and TRIM41 mRNA expression level in 30 LCLs (10 of each genotype) by qPCR analysis. Expression of BTNL9 could be detected at a moderate level in all celllines homozygous for the wild-type allele. In contrast, cell-lines heterozygous for the deletion expressed $B T N L 9$ at a significantly decreased amount, and in cell-lines homozygous for the deletion BTNL9 expression was almost not detectable (Figure 4A). In line, western-blot analysis revealed a strong decrease in protein expression levels in cell lines heterozygous or homozygous for the BTNL8_BTNL3-del allele (Figure 4B). In addition, a slight but not significant effect could be observed at the expression-level of TRIM7, which is located $\sim 200-\mathrm{kb}$ from the deletion (data not shown), implying that indeed it is due to a positional effect, although other possibilities cannot be ruled out yet.

The BTNL8*3 deletion CNV affects expression of several genes involved in immune response, cancer and developmental disorders

Next we looked at potential downstream targets by utilizing transcriptional data from the Illumina genomewide expression arrays of LCLs from 56 unrelated

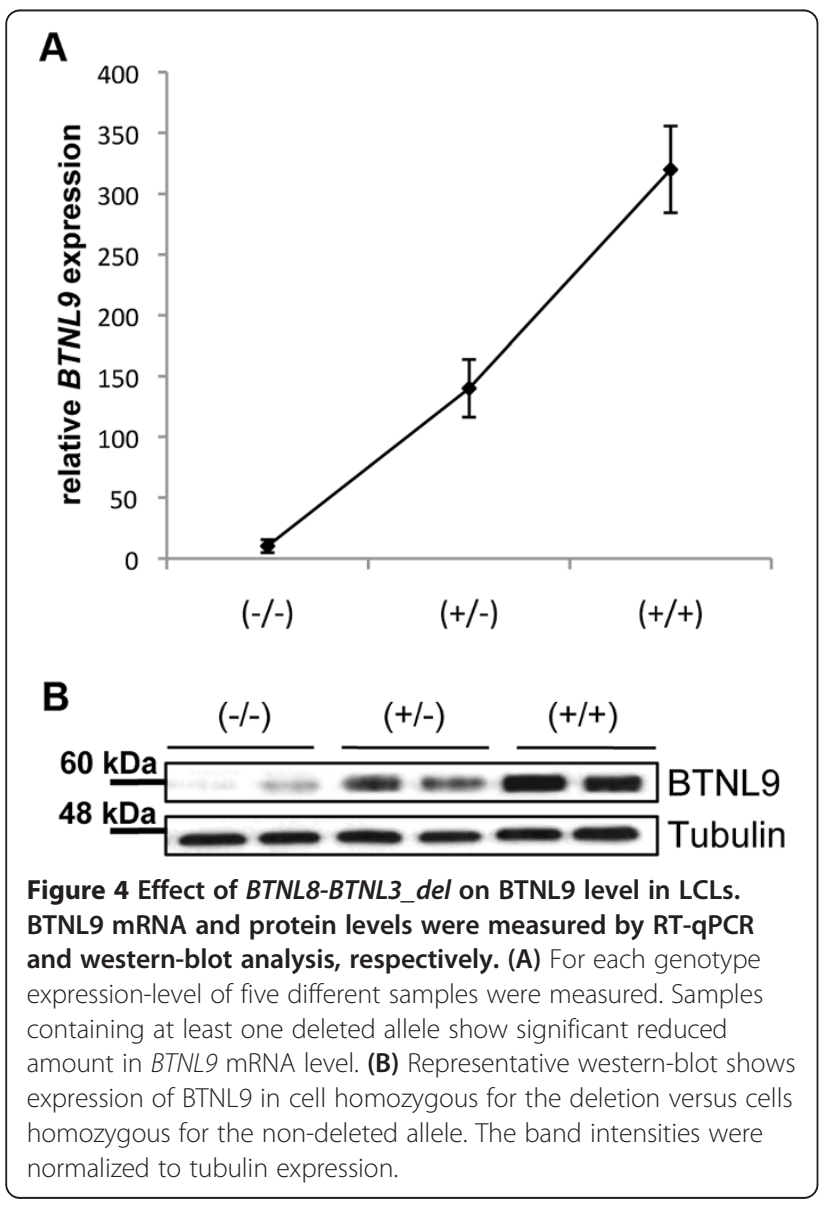

individuals, derived from CEU population [15]. Of the 56 cell-lines tested, 25 were homozygous for the insertion allele, 18 were heterozygous and 13 were homozygous for the deletion allele. In total, we found 20 genes showing a significant $(\mathrm{p}<0.05)$ differential expression according to the BTNL8/BTNL3 genotype (data not shown). Unfortunately, none of the genes deregulated by the CNV identified earlier, BTNL9 and TRIM7, were expressed at a detectable level. To verify the microarray data, we performed qPCR analysis on 10 LCLs homozygous for the wild-type allele and 10 LCLs homozygous for the deletion allele and tested all 20 genes found deregulated at the microarray. Of the 20 genes, nine could be validated as differentially expressed in LCLs homozygous for the BTNL8_BTNL3-del allele (Figure 5A).

In a next step, we submitted the data to Ingenuity Pathway Analysis (IPA) for functional classification and to check whether these genes interact with each other in regulatory networks and biological pathways. In addition we included the genes directly affected by the CNV; BTNL8, BTNL3 and BTNL9. The top functions mapped by IPA out of the 13 genes were: Hematopoiesis ( 3 genes, $\mathrm{p}<4.27 \mathrm{E}-05$ ), Immune Cell Trafficking (4 genes, $\mathrm{p}<4.27-$ 05), Hematological System Development and Function (5 genes, $\mathrm{p}<7.93 \mathrm{E}-05)$, Tissue Morphology (7 genes, $\mathrm{p}<$ 2.40E-04) Connective Tissue Development and Function (3 genes, $\mathrm{p}<3.08 \mathrm{E}-04)$. In addition, IPA formed one well-defined network, with function in cell morphology, cellular development and embryonic development, with nine mapped genes involved (Figure 5B). It should be noted that so far little is known about the function and operation mechanism of BTNL8, BTNL3 and BTNL9. Therefore they are not sufficiently well annotated for pathway analysis by Ingenuity Pathway.

\section{Discussion}

CNVs are thought to significantly contribute to populationbased adaptive evolution due to variability in expression levels. [16]. Especially recently emerged genes may have significantly contributed to evolutionary change and phenotypic adaptation in more recently diverged evolutionary lineages [17]. However, despite intensive work in the field, most CNVs and new resulting genes are so far, overall, rather poorly characterized at the functional level.

In this study, we provide a detailed functional analysis of a novel 56-kb deletion CNV involving two primate-specific genes of the $B T N L$ family, namely $B T N L 8$ and BTNL3, who share $80 \%$ homology in their coding sequence. The CNV has arisen in two 1.6-kb SD blocks of $98 \%$ identity, suggesting that the deletion occurred due to a NAHR event. CNVs arisen from NAHR tend to recur due to the unstable nature of the 


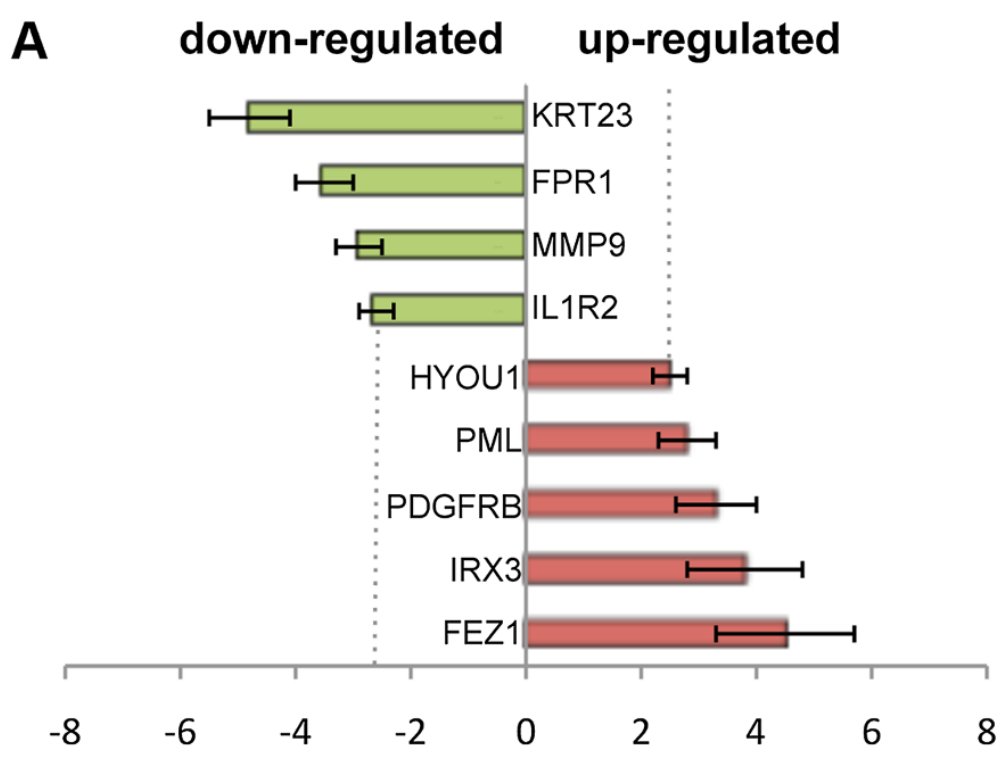

$\mathbf{B}$

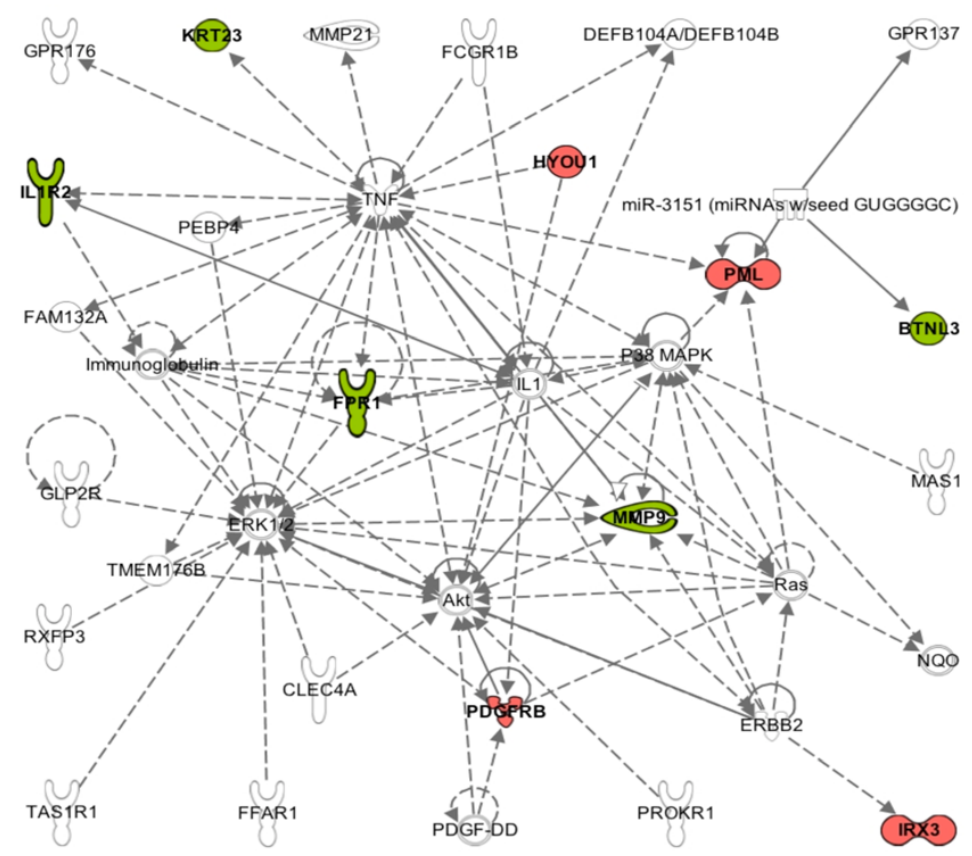

Figure 5 Differential expression of genes depending on BTNL8/BTNL3 genotype. (A) Nine genes could be confirmed by RT-qPCR analysis to be differentially expressed in LCLs homozygous for the BTNL8_BTNL3-del allele. Red, increased mRNA level; green, decreased mRNA level in LCLs homozygous for the CNV. (B) Network of genes formed by Ingenuity Pathway Analysis. Genes depicted in red (increased mRNA level) and green (decreased mRNA level) were confirmed by RT-qPCR analysis to be differentially expressed in LCLs homozygous for the CNV. Genes depicted in white were not found to be deregulated even though they are part of the same network.

large and highly identical SD, leading to variants of different haplotypes that share common endpoints. Multiple genomic disorders as Williams-Beuren syndrome, Charcot-Marie Tooth disease, schizophrenia, and developmental delay have been assigned to NAHR events between misaligned LCRs [18-21], making NAHR the most common mechanism mediating recurrent microdeletions and microduplications.

By carefully looking at SNPs data of the International HapMap project and through our own genotyping data, 
we found currently existing frequency-matched SNPs in LD $\left(\mathrm{r}^{2}>0.8\right)$ with the BTNL8*3 CNV in Eastern Asian, American and northern European populations. In contrast, no LD could be detected in African, Italian and Spanish populations. Especially African populations have been shown to be highly heterogeneous and to significantly vary in their haplotype structure and LD from other populations [22]. However, also southern European subgroups as Italian, Spanish and Greek, have been shown to differ in their haplotype composition from northern and western European populations [23]. This most likely results from large and constant migratory influences and admixture with other European and North African populations. These substantial differences between northern and southern European subgroups imply that many variations found through genome-wide association studies (GWAS) studies carried out on northern and western European population may not be replicated in southern European subgroups. Moreover, the absence of a tagging SNP in some populations has important implications for the interpretation of association studies. It stresses that unless a direct genotyping assay is applied for the $B T N L 8 * 3 \mathrm{CNV}$, its potential phenotypic impact may be overlooked in GWAS.

The BTNL8_BTNL3-del allele has been found at higher frequencies in European, East Asian and American populations, as compared to African, Oceanic and Middle Eastern populations. Ethnicity plays an important role in inter-individual variability of the immune system. Through recurrent exposure to different pathogens, ethnic groups have selected genetic adaptations that provide resistance or reduced susceptibility to infection [24] meaning that many times CNVs result in an advantageous phenotype for some populations [25,26]. An example of a reduction in copy number being beneficial has been suggested for the $\alpha$-globin locus, where it increases resistance to malaria infection and susceptibility to mild $\alpha$-thalassemia. In regions where malaria is endemic, the deleted form of the $\alpha$-globin gene can be found at an unusually high degree $[27,28]$. Other examples where the number of gene copies positively correlates with infection are FCGR3B and DEFB4 genes, which are associated with glomerulonephritis, and Crohn's disease, respectively $[29,30]$

Here we show that, in accordance with the partial deletion of these genes, the BTNL8 and BTNL3 proteins were not expressed in LCLs homozygous for the deletion, and they were found at a significantly reduced amount in LCLs carrying one deleted allele. RT-PCR analysis, sequencing and western blot analysis revealed a new BTNL8*3 fusion product without any alterations in the coding frame, suggesting the existence of a novel, functional protein. In addition, BTNL9, another member of the butyrophilin family, was down-regulated in LCLs carrying the BTNL8_BTNL3-del allele. However, the function of BTNL8, BTNL3 and BTNL9 is yet unknown; therefore, here we can only speculate what might be the consequence of the deletion.

The BTNL proteins belong to the butyrophilin (BTN) family. The eponymous BTN protein (BTN1A1) is a type I transmembrane glycoprotein whose expression is restricted to the mammary gland during lactation, where it is involved in the secretion of milk fat globules [31]. All BTN family members contain a signal peptide at their $\mathrm{N}$-terminus, two Ig-like-domains ( $\operatorname{IgV}$ and $\operatorname{IgC}$ ) and a transmembrane-domain. The extracellular domain shows structural similarities to those of the B7 family, a protein family of co-stimulatory molecules involved in $\mathrm{T}$ cell activation [32]. In contrast to B7 proteins, most BTN members harbour a cytoplasmic B30.2 domain at their C-terminus. Like the B7 protein family, several human and murine BTN and BTNL family members have been shown to control $\mathrm{T}$ cell response [33]. While the $B 7$ family of ligands and their receptors can regulate $T$ cell response either through their positive (e.g. B7-1, B72, ICOS) or negative (e.g. PD-L1, PD-L2, B7-H3, B7-H4) co-stimulatory molecules, BTNs so far only have been found to act through co-inhibition [34-37]. In addition, polymorphisms in the human gene encoding for BTNL2 have been linked to a growing number of inflammatory diseases, e.g. sarcoidosis, myositis and inflammatory bowel disease [38-40]. Moreover, human BTN2A1 has been shown to modulate immature dendritic cells (DC) by binding to Dendritic Cell-Specific Intercellular adhesion molecule-3-Grabbing Non-integrin (DC-SIGN) [41], and Btnl1 has been recently found to regulate interactions with intraepithelial $\gamma \delta \mathrm{T}$ lymphocytes in the murine small intestine by suppressing pro-inflammatory mediators of the NFKB pathway, such as IL-6, IL-15, CXCL1, and CCL4 [42]. In addition, many members of the B7-homolog (B7-H) family, such as PD-1 and CTLA-4, are expressed on tumour cells in various cancers, where they can be exploited by the cancerous cells to escape from immune destruction and impede B7 ongoing immune processes $[43,44]$. The presence of the deletion and the subsequent absence or reduced expression of the encoded proteins would allow a stronger response against tumour cells, implying that the BTNL8_BTNL3-del allele could act as a positive modulator of anti-tumour immunity. However, co-inhibitory molecules such as CTLA-4, PD-1 and BTLA have been shown to be crucial for the prevention of autoimmunity and polymorphism or deficiency of these molecules are associated with genetic susceptibility to autoimmune diseases in human and mice [45]. Followup studies on the BTNL proteins will shed more light on their function in autoimmune diseases and cancer.

$B T N L 8$ and $B T N L 3$ are primate-specific genes, while $B T N L 9$ has a clear ortholog in mice. Human BTNL8 and 
BTNL3 expression is primarily restricted to tissues of the digestive tract and at a lower level to spleen, thymus and lung. In addition BTNL3 is expressed in neutrophils and BTNL8 in eosinophils and at a reduced amount in neutrophils. In contrast, BTNL9 is mainly expressed in B cells and lymphoid organs, e.g. thymus, spleen, bone marrow and lymph nodes $[46,47]$. Due to the high sequence homology and the similar expression-profile of BTNL8 and BTNL3 it is possible that the new BTNL8*3 fusion-protein compensates for the BTNL8 and BTNL3 wild-type proteins. However, more information is needed about the functions of BTNL8, BTNL3 and BTNL8*3 and the pathways they are involved in. Since almost all members of the BTN and BTNL families are highly expressed in the intestine [46], it has been postulated that these proteins act through a combined immunosuppressive effect, rather than a big impact of individual molecules [48]. Therefore, it would be interesting to check for the consequences of the CNV in diseases associated with polymorphisms in BTN and BNTL genes, e.g. BTNL2 in Crohn's disease or ulcerative colitis. Nevertheless, a review of the published GWAS data in these two disorders did not reveal an association with the potential tagging SNPs for the deleted allele (rs2387715, rs4700772 or rs10063135), although these SNPs are present in the affy 6.0 (rs2387715) and Illumina Omni 1 and human $1 \mathrm{M}$ arrays (rs4700772 and rs10063135. There is, nevertheless, a replicated association on Chron's disease to the $5 \mathrm{q} 35$ region around $C P E B 4$ gene $[49,50]$, about $3 \mathrm{Mb}$ away from the deletion.

Interestingly, when investigating gene expression changes with regard to the BTNL8_BTNL3-del allele, we found TNF and the ERK1/AKT pathway to be central hubs of the network influenced by the deletion CNV in LCLs. TNF, ERK1 and AKT are important players in signal transduction pathways and key components of the immune response in humans and even a slight deregulation of those proteins might have an important impact in the response to pathogens. However, even though the HapMap repository represents a fantastic source for genetic studies, the analysis was limited due to the use of LCLs, which might not be the main cell-type where the BTNL8*3 CNV affects expression levels, since BTNL3 and BTNL8 are predominantly expressed in the digestive tract. Follow-up studies using other cell types will be needed.

\section{Conclusion}

In summary, we provide a broad, functional analysis of a common deletion variant at several levels. We demonstrate the existence of a new fusion-protein, implying functional consequences of the CNV. Moreover, we proof structural changes at the immediate neighborhood of the CNV likely due to a position effect, as well as overall changes in the general expression-level of several genes involved in immune regulation and proliferation. Although the exact molecular function of BTNL8, BTNL3 and BTNL9 remains unknown, the high frequency of the deletion in some populations, its structural homology to B7 proteins and its tissue distribution make the $\mathrm{CNV}$ a potentially interesting candidate for diseases associated with infection and inflammation, especially in the gut.

\section{Methods \\ Samples}

1,103 samples from the International HapMap Phase III project, 1,007 individuals from the CEPH-HDGP cohort, and 2,000 Spanish samples were genotyped [51,52]. The samples consisted of 120 individuals from Utah with northern and western European ancestry (CEU); 90 Han Chinese from Bejing (CHB); 90 Japanese from Tokyo (JPT); 120 Yoruba form Ibadan, Nigeria (YRI); 43 individuals with African ancestry from Southwest USA (ASW); 100 Han Chinese from Denver, Colorado (CHD); 100 Gujarati Indians from Houston, Texas (GIH); 100 Luhya from Webuya, Kenya (LWK); 90 of Mexican ancestry from Los Angeles, California (MEX); 150 Maasai from Kinyawa, Kenya (MKK); 100 Toscans from Italy (TSI); and an independent control cohort of 2,000 Spanish unrelated individuals. The analysis from the CEPH-HGDP includes individuals from 51 different populations and excludes samples previously identified as duplicates as well as the genotypically abnormal samples 770 and 980 [53]. Individual genotypes are provided in Additional file 1: Table S1 and Table S2.

\section{Genotyping and sequence analysis}

To identify possible CNVs, we used the Human Genome CGH Microarray Kit (Agilent 244 k aCGH microarray kit) with covers the genome with a $10-\mathrm{kb}$ resolution between probes. aCGH was performed according to manufacturer's protocol and as described previously [54]. Results obtained for the 5q35.3 region were confirmed by MLPA analysis and long range PCR analysis. Sequences of MLPA probes used in the study are listed in Additional file 1: Table S5.

\section{Statistical analysis}

Linkage disequilibrium was calculated and visualized with Haploview. Statistical differences of allelic distributions among populations were assessed performing a chi-square test.

\section{PCR genotyping assay}

Two allele-specific PCR assays using fluorescent oligonucleotides, were designed to distinguish non-deletion and deletion alleles based on primer sequences: non-del forward: 5'-(HEX)GGCACAACCCAGAACAAAGT-3', 
non-del-reverse: 5' -TGAGAACCAAAATGAGCACAA3', del-forward: 5' -TTCCATGAACACCACCAAGA-3' and del-reverse: $5^{\prime}$-(HEX)GACACAGGAGTGTGCAA GGT-3'. DNA from HapMap and CEPH-HGDP samples was available in the lab. Quality of DNA was checked on a 1\% agarose gel prior PCR and samples with degraded DNA were excluded from the analysis. PCR was carried out in a GeneAmp PCR System (Applied Biosystems) using $100 \mathrm{ng}$ of genomic DNA/reaction. Conditions consist of an initial denaturation step of $1 \mathrm{~min}$ at $95^{\circ} \mathrm{C}$, 32 cycles of $30 \mathrm{~s}$ at $95^{\circ} \mathrm{C}$ denaturation, $30 \mathrm{~s}$ annealing at $62.5^{\circ} \mathrm{C}$ and $25 \mathrm{~s}$ elongation at $72^{\circ} \mathrm{C}$ and a final extension step at $72^{\circ} \mathrm{C}$ for $25 \mathrm{~min}$. Non-deletion primers were located inside the deleted sequence and generated a 420 bp PCR product. Deletion primers amplified across the deletion breakpoints and resulted in a 340-bp product.

\section{Real-Time PCR analysis}

Total mRNA was extracted from cells using the miRNA easy Kit (Qiagen), samples were treated with DNase I (Qiagen) for $15 \mathrm{~min}$ and 1 to $2 \mu \mathrm{g}$ of RNA was reverse transcribed using the Superscript VILO kit (Invitrogen) according to the manufacturer's protocol. Real time PCR was carried out using the Light cycler 480 from Roche. The PCR reaction contained $35 \mathrm{ng}$ of cDNA, $10 \mathrm{pmol}$ of each of the specific primers and $5 \mu \mathrm{l}$ SYBR Green master mix in a final reaction volume of $10 \mu \mathrm{l}$. All reactions were performed in triplicates. Thermal-cycling conditions consisted of an initial denaturation of $10 \mathrm{~min}$ at $98^{\circ} \mathrm{C}, 40 \mathrm{cy}-$ cles of $15 \mathrm{~s}$ at $95^{\circ} \mathrm{C}$ denaturation, $15 \mathrm{~s}$ annealing, and $18 \mathrm{~s}$ elongation at $72^{\circ} \mathrm{C}$, and final extension at $72^{\circ} \mathrm{C}$ for $10 \mathrm{~min}$. The optimal annealing temperature was determined for each oligonucleotide-pair individually. Cumulative fluorescence was measured after each of the 40 cycles. Product specific amplification was confirmed by melting curve analysis. Oligonucleotide sequences used for quantification are listed in Additional file 1: Table S6. Relative quantification of gene expression was determined by the construction of a relative expression calibration curve using serial dilutions of $A C T B$ and $G A P D H$ as a positive control.

\section{Allele-specific expression profiling}

RNA extraction, DNase treatment, first-strand cDNA synthesis and qPCR were done as described above with BTNL8 5' 'TTTGGCATTGTTGGACTGAA-3' as forward primer and BTNL3 5'-ACACTCCCACATACCA CCCT-3' and BTNL8 5'-TCCTTCCTCCTGTCCACA $\mathrm{TC}-3^{\prime}$ reverse primers.

\section{Western blot}

Cell lysates were prepared as previously described [55]. Briefly, equal amounts of proteins (300-350 $\mu \mathrm{g})$ were resolved by NuPAGE (4-12\%; Invitrogen) and transferred to nitrocellulose membranes. Proteins were then blocked by incubation in $10 \%$ dry milk in TBST $(0.1 \%$ Tween-20 in TBS) and probed with the indicated Antibody. Blots were then developed by enhanced chemiluminescence (ECL; Amersham).

\section{Antibodies}

A polyclonal BTNL3 antibody was generated to peptides YIQHAMYDEEKGTPI, PPSTPPTRVGVFLDYE and YW VLRLTTEHLYFTF, corresponding to the cytoplasmic region of human BTNL3, according to standard procedures. Briefly, the three synthetic peptides were independently keyhole limpet hemocyanin (KLH)-conjugated, pooled, and injected into two female New Zealand White rabbits for antisera production. Pre-immune serum was collected before immunization and was used as a negative control. Antisera collected from the immunized rabbits were verified for reactivity by ELISA against the original peptides and the human BTNL3 protein. Rabbit BTNL9 antibody was purchased from Abcam (ab87049). Mouse Tubulin antibody was purchased from Santa Cruz (sc-5286).

\section{Expression analysis and Ingenuity Pathway Analysis software} Microarray expression data were obtained from Stranger et al., [15] and analyzed for differences in the expression level regarding the BTNL8/BTNL3 genotype. The list of genes found to be influenced by the BTNL $8 * 3$ deletion CNV was submitted to Ingenuity Pathway Analysis (IPA; Ingenuity ${ }^{\circ}$ Systems, Redwood City, CA, http://www.ingenuity.com) to identify common biological pathways. In addition, gene expression values were included in the input to identify up and down regulated genes in the pathways.

\section{Additional file}

Additional file 1: Figure S1. Protein alignment of BTNL8 and BTNL3. BTNL8 and BTNL3 share $68.5 \%$ similarity in their amino-acid sequences. Segmental duplication, where cross-over occurred, is highlighted in yellow. Table S1. Individual genotyping HapMap. Table S2. Individual genotyping CEPH-HGDP. Table S3. Frequency of deletion by continental groups. Table S4. CEPH-HGDP genotype frequencies by geographic location. Table S5. Sequences of MLPA probes. Table S6. Sequences of oligonucleotides.

\section{Competing interests}

The authors declare that they have no competing interests.

\section{Authors' contributions}

JA, EM and XE designed the study. JA and SV performed all experimental work. JA wrote the manuscript. JA and RR analyzed the data. RR performed the statistical analysis. JR and JJC collected data and participated in their interpretation. All authors read and approved the final manuscript.

\section{Acknowledgements of research support}

The project was supported by grants from Obra Social La Caixa to J.A.; the Ramon y Cajal program to R.R.; the Spanish Ministry of Science and Innovation, Instituto de Salud Carlos III with the grants "Registro BASICMAR" Funding for Research in Health (PI051737), "GWALA project" from Fondos de Investigación Sanitaria ISC III (PI10/02064); and Fondos FEDER/EDRF Red de Investigación Cardiovascular (RD12/0042/0020) and 
the Fundació la Marató TV3 with the grant "GOD's project. Genestroke Consortium" (76/C/2011) to J.R. and J.J.C.; the Spanish Ministry of Science and Innovation (SAF2008-00357to NOVADIS project); the European Commission (ENGAGE project and grant agreement HEALTH-F4-2007201413); and the Generalitat de Catalunya (Agència de Gestió d'Ajuts Universitaris i de Recerca-AGAUR) to X.E.

\section{Author details}

${ }^{1}$ Bioinformatics and Genomics Program, Centre for Genomic Regulation (CRG), Barcelona 08003, Spain. ${ }^{2}$ Universitat Pompeu Fabra (UPF), Barcelona 08003, Spain. ${ }^{3}$ Centro de Investigación Biomédica en Red en Epidemiología y Salud Pública (CIBERESP), Barcelona 08003, Spain. ${ }^{4}$ Hospital del Mar Medical Research Institute (IMIM), Barcelona 08003, Spain. ${ }^{5}$ Neurology Department, Neuvovascular Research Group, Institut Hospital del Mar d'Investigacions Mèdiques (IMIM), Barcelona 08003, Spain. ${ }^{6}$ Universitat Autònoma de Barcelona (UAB), Barcelona 08003, Spain.

Received: 29 January 2013 Accepted: 21 May 2013 Published: 6 July 2013

\section{References}

1. Mills RE, Walter K, Stewart C, Handsaker RE, Chen K, Alkan C, Abyzov A Yoon SC, Ye K, Cheetham RK, et al: Mapping copy number variation by population-scale genome sequencing. Nature 2011, 470:59-65.

2. Pinto D, Pagnamenta AT, Klei L, Anney R, Merico D, Regan R, Conroy J, Magalhaes TR, Correia C, Abrahams BS, et al: Functional impact of global rare copy number variation in autism spectrum disorders. Nature 2010, 466:368-372.

3. Girirajan S, Campbell CD, Eichler EE: Human copy number variation and complex genetic disease. Annu Rev Genet 2011, 45:203-226.

4. Orange JS, Glessner JT, Resnick E, Sullivan KE, Lucas M, Ferry B, Kim CE, Hou C, Wang F, Chiavacci R, et al: Genome-wide association identifies diverse causes of common variable immunodeficiency. J Allergy Clin Immunol 2011, 127:1360-1367.

5. Vacic V, McCarthy S, Malhotra D, Murray F, Chou HH, Peoples A, Makarov V Yoon S, Bhandari A, Corominas R, et al: Duplications of the neuropeptide receptor gene VIPR2 confer significant risk for schizophrenia. Nature 2011, 471:499-503.

6. She X, Liu G, Ventura M, Zhao S, Misceo D, Roberto R, Cardone MF, Rocchi M, Green ED, Archidiacano N, Eichler EE: A preliminary comparative analysis of primate segmental duplications shows elevated substitution rates and a great-ape expansion of intrachromosomal duplications. Genome Res 2006, 16:576-583.

7. Jiang $Z$, Tang $H$, Ventura $M$, Cardone MF, Marques-Bonet $T$, She $X$, Pevzner PA, Eichler EE: Ancestral reconstruction of segmental duplications reveals punctuated cores of human genome evolution. Nat Genet 2007, 39:1361-1368.

8. Bailey JA, Gu Z, Clark RA, Reinert K, Samonte RV, Schwartz S, Adams MD, Myers EW, Li PW, Eichler EE: Recent segmental duplications in the human genome. Science 2002, 297:1003-1007.

9. Liu P, Lacaria M, Zhang F, Withers M, Hastings PJ, Lupski JR: Frequency of nonallelic homologous recombination is correlated with length of homology: evidence that ectopic synapsis precedes ectopic crossingover. Am J Hum Genet 2011, 89:580-588.

10. Armengol L, Villatoro S, Gonzalez JR, Pantano L, Garcia-Aragones M, Rabionet $R$, Caceres $M$, Estivill $X$ : Identification of copy number variants defining genomic differences among major human groups. PLoS One 2009, 4:e7230.

11. Gokcumen $\mathrm{O}$, Babb PL, Iskow RC, Zhu Q, Shi X, Mills RE, Ionita-Laza I, Vallender EJ, Clark AG, Johnson WE, Lee C: Refinement of primate copy number variation hotspots identifies candidate genomic regions evolving under positive selection. Genome Biol 2011, 12:R52.

12. Kim JI, Ju YS, Park H, Kim S, Lee S, Yi JH, Mudge J, Miller NA, Hong D, Bell $\mathrm{CJ}$, et al: A highly annotated whole-genome sequence of a Korean individual. Nature 2009, 460:1011-1015.

13. Henrichsen $\mathrm{CN}$, Chaignat E, Reymond A: Copy number variants, diseases and gene expression. Hum Mol Genet 2009, 18:R1-R8.

14. De S, Babu MM: Genomic neighbourhood and the regulation of gene expression. Curr Opin Cell Biol 2010, 22:326-333.
15. Stranger BE, Forrest MS, Dunning M, Ingle $C E$, Beazley $C$, Thorne $N$, Redon R, Bird CP, de Grassi A, Lee C, et al: Relative impact of nucleotide and copy number variation on gene expression phenotypes. Science 2007, 315:848-853.

16. Kaessmann $\mathrm{H}$ : Origins, evolution, and phenotypic impact of new genes. Genome Res 2010, 20:1313-1326.

17. Metzker ML: Sequencing technologies - the next generation. Nat Rev Genet 2010, 11:31-46.

18. Merla G, Brunetti-Pierri N, Micale L, Fusco C: Copy number variants at Williams-Beuren syndrome 7q11.23 region. Hum Genet 2010, 128:3-26

19. Szigeti K, Lupski JR: Charcot-Marie-Tooth disease. Eur J Hum Genet 2009, 17:703-710.

20. Malhotra D, McCarthy S, Michaelson JJ, Vacic V, Burdick KE, Yoon S, Cichon S, Corvin A, Gary S, Gershon ES, et al: High frequencies of de novo CNVs in bipolar disorder and schizophrenia. Neuron 2011, 72:951-963.

21. Vissers LE, Stankiewicz P: Microdeletion and microduplication syndromes. Methods Mol Biol 2012, 838:29-75

22. Ptak SE, Voelpel K, Przeworski M: Insights into recombination from patterns of linkage disequilibrium in humans. Genetics 2004, 167:387-397.

23. A map of human genome variation from population-scale sequencing. Nature 2010, 467:1061-1073.

24. Campbell MC, Tishkoff SA: African genetic diversity: implications for human demographic history, modern human origins, and complex disease mapping. Annu Rev Genomics Hum Genet 2008, 9:403-433.

25. Xue Y, Sun D, Daly A, Yang F, Zhou X, Zhao M, Huang N, Zerjal T, Lee C, Carter NP, et al: Adaptive evolution of UGT2B17 copy-number variation. Am J Hum Genet 2008, 83:337-346.

26. Cook EH Jr, Scherer SW: Copy-number variations associated with neuropsychiatric conditions. Nature 2008, 455:919-923.

27. Flint J, Hill AV, Bowden DK, Oppenheimer SJ, Sill PR, Serjeantson SW, BanaKoiri J, Bhatia K, Alpers MP, Boyce AJ, et al: High frequencies of alphathalassaemia are the result of natural selection by malaria. Nature 1986, 321:744-750.

28. Kwiatkowski DP: How malaria has affected the human genome and what human genetics can teach us about malaria. Am J Hum Genet 2005, 77:171-192.

29. Aitman TJ, Dong R, Vyse TJ, Norsworthy PJ, Johnson MD, Smith J, Mangion J, Roberton-Lowe C, Marshall AJ, Petretto E, et al: Copy number polymorphism in Fcgr3 predisposes to glomerulonephritis in rats and humans. Nature 2006, 439:851-855.

30. Bentley RW, Pearson J, Gearry RB, Barclay ML, McKinney C, Merriman TR, Roberts RL: Association of higher DEFB4 genomic copy number with Crohn's disease. Am J Gastroenterol 2010, 105:354-359.

31. Robenek H, Hofnagel O, Buers I, Lorkowski S, Schnoor M, Robenek MJ, Heid $H$, Troyer D, Severs NJ: Butyrophilin controls milk fat globule secretion. Proc Natl Acad Sci USA 2006, 103:10385-10390.

32. Linsley PS, Peach R, Gladstone P, Bajorath J: Extending the B7 (CD80) gene family. Protein Sci 1994, 3:1341-1343.

33. Abeler-Dorner L, Swamy M, Williams G, Hayday AC, Bas A: Butyrophilins: an emerging family of immune regulators. Trends Immunol 2012, 33:34-41.

34. Yamazaki T, Goya I, Graf D, Craig S, Martin-Orozco N, Dong C: A butyrophilin family member critically inhibits T cell activation. J Immunol 2010, 185:5907-5914

35. Smith IA, Knezevic BR, Ammann JU, Rhodes DA, Aw D, Palmer DB, Mather $\mid H$, Trowsdale J: BTN1A1, the mammary gland butyrophilin, and BTN2A2 are both inhibitors of T cell activation. J Immunol 2010, 184:3514-3525.

36. Nguyen T, Liu XK, Zhang Y, Dong C: BTNL2, a butyrophilin-like molecule that functions to inhibit T cell activation. J Immunol 2006, 176:7354-7360.

37. Arnett HA, Escobar SS, Gonzalez-Suarez E, Budelsky AL, Steffen LA, Boiani N, Zhang M, Siu G, Brewer AW, Viney JL: BTNL2, a butyrophilin/B7-like molecule, is a negative costimulatory molecule modulated in intestinal inflammation. J Immunol 2007, 178:1523-1533.

38. Wijnen PA, Voorter CE, Nelemans PJ, Verschakelen JA, Bekers O, Drent M: Butyrophilin-like 2 in pulmonary sarcoidosis: a factor for susceptibility and progression? Hum Immunol 2011, 72:342-347. 
39. Scott AP, Laing NG, Mastaglia F, Needham M, Walter MC, Dalakas MC, Allcock RJ: Recombination mapping of the susceptibility region for sporadic inclusion body myositis within the major histocompatibility complex. J Neuroimmunol 2011, 235:77-83.

40. Johnson CM, Traherne JA, Jamieson SE, Tremelling M, Bingham S, Parkes M, Blackwell JM, Trowsdale J: Analysis of the BTNL2 truncating splice site mutation in tuberculosis, leprosy and Crohn's disease. Tissue Antigens 2007, 69:236-241.

41. Malcherek G, Mayr L, Roda-Navarro P, Rhodes D, Miller N, Trowsdale J: The B7 homolog butyrophilin BTN2A1 is a novel ligand for DC-SIGN. J Immunol 2007, 179:3804-3811.

42. Bas A, Swamy M, Abeler-Dorner L, Williams G, Pang DJ, Barbee SD, Hayday AC: Butyrophilin-like 1 encodes an enterocyte protein that selectively regulates functional interactions with T lymphocytes. Proc Natl Acad Sci USA 2011, 108:4376-4381.

43. Martin-Orozco N, Dong C: Inhibitory costimulation and anti-tumor immunity. Semin Cancer Biol 2007, 17:288-298.

44. Driessens G, Kline J, Gajewski TF: Costimulatory and coinhibitory receptors in anti-tumor immunity. Immunol Rev 2009, 229:126-144.

45. Watanabe $\mathrm{N}$, Nakajima $\mathrm{H}$ : Coinhibitory molecules in autoimmune diseases. Clin Dev Immunol 2012, 2012:269756.

46. Arnett HA, Escobar SS, Viney JL: Regulation of costimulation in the era of butyrophilins. Cytokine 2009, 46:370-375.

47. Shibui A, Tsunoda T, Seki N, Suzuki Y, Sugano S, Sugane K: Cloning, expression analysis, and chromosomal localization of a novel butyrophilin-like receptor. J Hum Genet 1999, 44:249-252.

48. Cubillos-Ruiz JR, Conejo-Garcia JR: It never rains but it pours: potential role of butyrophilins in inhibiting anti-tumor immune responses. Cell Cycle 2011, 10:368-369.

49. Franke A, McGovern DP, Barrett JC, Wang K, Radford-Smith GL, Ahmad T, Lees CW, Balschun T, Lee J, Roberts R, et al: Genome-wide meta-analysis increases to 71 the number of confirmed Crohn's disease susceptibility loci. Nat Genet 2010, 42:1118-1125.

50. Jostins L, Ripke S, Weersma RK, Duerr RH, McGovern DP, Hui KY, Lee JC, Schumm LP, Sharma Y, Anderson CA, et al: Host-microbe interactions have shaped the genetic architecture of inflammatory bowel disease. Nature 2012, 491:119-124

51. Cann HM, de Toma C, Cazes L, Legrand MF, Morel V, Piouffre L, Bodmer J, Bodmer WF, Bonne-Tamir B, Cambon-Thomsen A, et al: A human genome diversity cell line panel. Science 2002, 296:261-262.

52. A haplotype map of the human genome. Nature 2005, 437:1299-1320.

53. Rosenberg NA: Standardized subsets of the HGDP-CEPH Human Genome Diversity Cell Line Panel, accounting for atypical and duplicated samples and pairs of close relatives. Ann Hum Genet 2006, 70:841-847.

54. de Cid R, Riveira-Munoz E, Zeeuwen PL, Robarge J, Liao W, Dannhauser EN, Giardina E, Stuart PE, Nair R, Helms C, et al: Deletion of the late cornified envelope LCE3B and LCE3C genes as a susceptibility factor for psoriasis. Nat Genet 2009, 41:211-215.

55. Minones-Moyano E, Porta S, Escaramis G, Rabionet R, Iraola S, Kagerbauer B, Espinosa-Parrilla Y, Ferrer I, Estivill X, Marti E: MicroRNA profiling of Parkinson's disease brains identifies early downregulation of miR-34b/c which modulate mitochondrial function. Hum Mol Genet 2011, 20:3067-3078.

\section{Submit your next manuscript to BioMed Central and take full advantage of:}

- Convenient online submission

- Thorough peer review

- No space constraints or color figure charges

- Immediate publication on acceptance

- Inclusion in PubMed, CAS, Scopus and Google Scholar

- Research which is freely available for redistribution

Submit your manuscript at www.biomedcentral.com/submit 